\title{
Realization of Structural Color by Direct Laser Write Technique in Photoresist
}

\author{
Vygantas MIZEIKIS $^{* 1}$, Vytautas PURLYS ${ }^{* 2}$, Ričardas BUIVIDAS ${ }^{* 3}$ and Saulius JUODKAZIS ${ }^{* 3}$ \\ ${ }^{* 1}$ Research Institute of Electronics, Shizuoka University, \\ 3-5-3-1 Johoku, Naka-ku, Hamamatsu 432-8561, Japan \\ E-mail:dvmzks@ipc.shizuoka.ac.jp \\ ${ }^{* 2}$ Laser Research Center, Department of Quantum Electronics, \\ Vilnius University, Vilnius, Lithuania \\ ${ }^{* 3}$ Centre for Micro-Photonics, Swinburne University of Technology, Melbourne, Australia
}

\begin{abstract}
We report realization of structural color in 3D woodpile photonic crystal structures fabricated by femtosecond direct laser write (DLW) lithography in photoresist. The structural color in the fabricated samples was obtained due to spectral regions with high optical reflectivity associated with higher photonic bands well above the fundamental (lowest frequency) photonic stop gap, which allowed realization of visible colors without the need to reduce the lattice period. This advantage facilitates easier and faster DLW fabrication, and can be exploited for realization of structural color materials for various applications, for example optical environmental sensing.

DOI: $10.2961 / \mathrm{jlmn} .2014 .01 .0009$
\end{abstract}

Keywords: structural color, photonic crystals, direct laser write technique

\section{Introduction}

Structural color arises in periodic materials due to complex interplay between multiple light interference and diffraction as well as random scattering on the wavelength scale. Many natural systems, such as opals, wings of butterflies and bugs, scales of fish, etc., exhibit bright, angleindependent structural color[1]. There is a considerable interest in achieving pigment-free, non-fading, bright structural colors with artificially micro- and nano-structured materials[2], but this is a challenging task, because 3D patterning of materials with sub-micron resolution is required in order to achieve structural color at visible wavelengths. Here, we describe realization of controllable structural color in 3D woodpile architecture[3] photonic crystals[4] fabricated in photoresist by femtosecond Direct Laser Write (DLW) technique[5]. The color of fabricated samples exhibits gradual change from red to green when power of the writing laser beam and lattice parameters of woodpile structure are varied. This behaviour cannot be associated with optical reflectance due to fundamental photonic stop gaps (PSG), since woodpile lattice period and other parameters gaps indicate the fundamental stop gap at nearinfrared wavelength near $\lambda \approx 1.5 \mu \mathrm{m}$. The color is therefore attributed to interplay between higher-order PSG[6] reflectance, diffraction, and random scattering on structural imperfections. These results indicate the possibility to realize structural color by DLW technique in photoresists without the need to severely downscale the lattice period, which would allow fast realization of porous dielectrics with pronounced, angle-independent structural colors for various applications, such as optical sensing[7].

\section{Experimental details}

Optical setup used for the DLW experiments is schematically depicted in Fig. 1(a). The laser source was a Mai Tai (Spectra Physics) femtosecond laser with a temporal pulse length (at the focus) of $100 \mathrm{fs}$, a central wavelength of $\lambda=800 \mathrm{~nm}$, and a repetition rate of $80 \mathrm{MHz}$. The laser beam was attenuated by an acousto-optical modulator (AOM) and focused into the bulk of photoresist film by an oil-immersion microscope objective lens with a numerical aperture $\mathrm{NA}=1.35$. Laser writing was facilitated by translating the sample mounted on a computer-
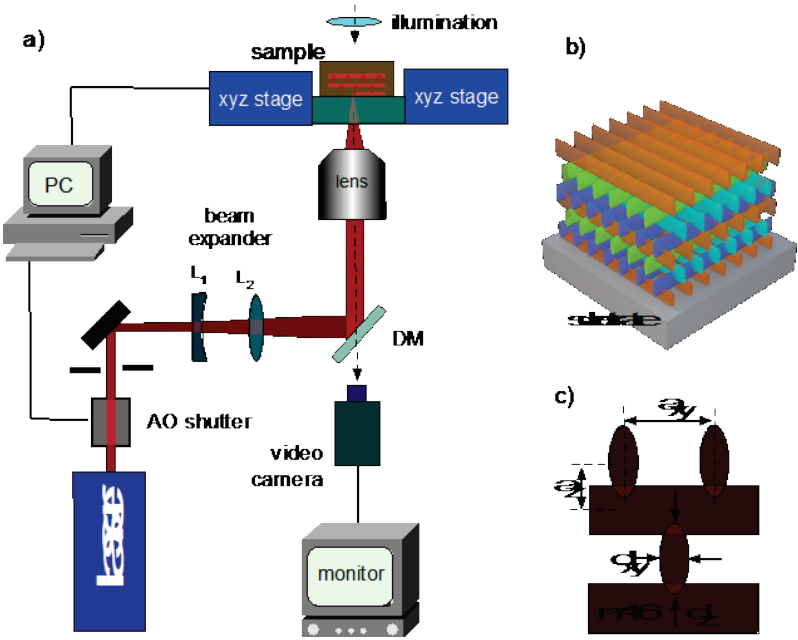

c)

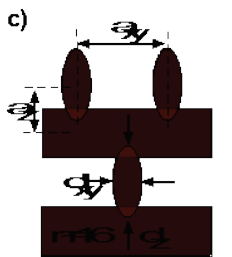

Fig. 1 (a) DLW experimental setup, (b) woodpile structure on a glass substrate, (c) main parameters of woodpile architecture. 
controlled three-dimensional translation stage along a preset trajectory with a linear translation speed typically set in the range of $50-100 \mu \mathrm{m} / \mathrm{s}$. The DLW setup (except the laser) is assembled on a massive granite base plate to prevent vibrations and thermal position drift during the fabrication, and allows in situ observation of the fabrication process by a video camera.

The samples consisted of hybrid organic-inorganic photoresist specially developed for 3D laser lithography [8] and often referred as SZ2080. The resist was drop-cast and dried on a microscope cover glass substrates (Matsunami) with thickness of about $180 \mu \mathrm{m}$, which allowed focusing of the laser beam into photoresist through the substrate as shown in Fig. 1(b). The exposed samples were developed in 1-propanol:isopropanol (50:50) solution, rinsed in ethanol, and dried in a super-critical $\mathrm{CO}_{2}$ dryer (JCPD-5, JEOL) to prevent destruction of fragile, finely-patterned, highly-porous photoresist structures by capillary forces[9] common during conventional drying.

3D woodpile architecture [3] and its main parameters are explained in Fig 1(b,c). In all samples, in-plane and vertical lattice periods, $a_{x y}$ and $a_{z}$, respectively, are kept at the fixed ratio of $a_{z}=0.35 a_{x y}$ in order to maintain facecentered cubic (fcc) unit cell. Cross sectional shape of woodpile rods is elliptical, with minor and major diameters $d_{x y}$ and $d_{z}$ related as $d_{z} / d_{x y} \approx 2.8$ due to elongation of twophoton point-spread function of the focused laser beam[10].

Structural characterization of the fabricated samples was performed using optical as well as Scanning Electron Microscopy (SEM). Optical reflectivity spectra of the samples were measured in the visible spectral range using a home-made micro-reflectivity setup based on a modified optical microscope, and in the near-infrared (NIR) spectral range using Fourier-Transform Infra-Red (FTIR) microspectrometer (Nicolet Nexus with a Continuum infrared microscope).

\section{Results and discussion}

Illustration of apparent structural color in the fabricated structures is given in Fig. 2(a). The Figure shows optical microscopy images (taken in reflection with NA $=0.3$ imaging lens) of a set of woodpile photonic crystals having the same footprint of $(25 \times 25) \mu \mathrm{m}^{2}$ and the same number of layers $\mathrm{N}=20$ along the stacking direction. The bottom-left corner of the image represents the origin point featuring the structure having in-plane period of $a_{x y}=700 \mathrm{~nm}$ and fabricated at the average writing laser power of $P_{\text {las }}=1.9 \mathrm{~mW}$. Proceeding from the origin along the arrows indicated in the Figure, $a_{x y}$ and $P_{\text {las }}$ are increased with increments of $50 \mathrm{~nm}$ and $0.1 \mathrm{~mW}$, respectively. Below we will refer to various structures by denoting pairs of parameters $\left(a_{x y}, P_{l a s}\right)$. As can be seen from the image, some of the structures in the matrix exhibit bright visible colors, which vary with the lattice period and the laser power. The apparent color correlates well with the optical reflectivity spectra. This is illustrated by the measured reflectivties of three selected structures (denoted by numbers 1, 2, 3 in Fig. 2 (a)), shown in Fig. 2(b). As can be seen, the reflectance bands exhibit red shift with woodpile lattice period and the writing laser power (i.e., dielectric filling fraction), in qualitative agreement with Maxwell's scaling behavior typical for photonic crystals[4]. This circumstance indicates that the color is a)

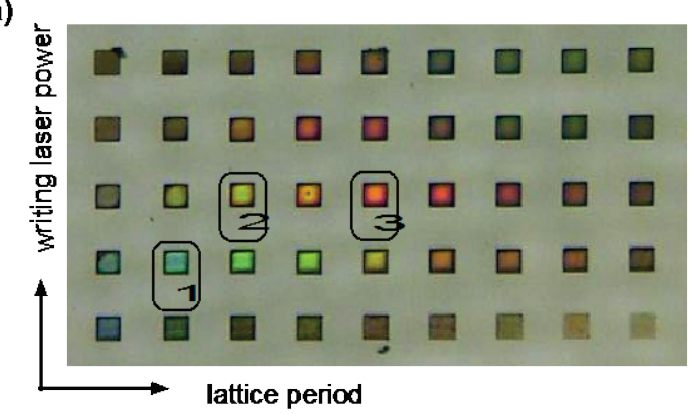

b)

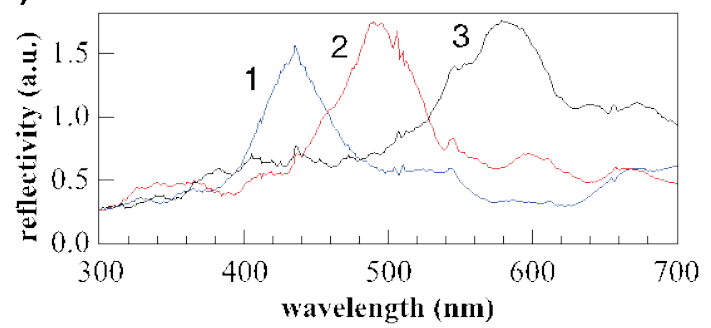

Fig. 2 (a) Optical microscopy images of several woodpile structures for various lattice periods and DLW laser power levels, (b) visible optical reflectivity spectra of three selected structures from (a).

related to PSG in the fabricated structures. This relation will be discussed below.

We stress here, that optical images and the corresponding spectra were acquired in an optical microscope using lens with numerical aperture $\mathrm{NA}=0.3$ for sample illumination and imaging. Under these circumstances, the images and spectra represent statistical convolution of incidence and detection angles within the approximate angular range of $\pm 17^{0}$ with respect to the woodpile layer stacking direction (normal to the woodpile layers). The clarity, with which colors are observed visually and spectrally, indicates their independence of the observation/illumination direction in the given angular range. This angular invariance likely originates from disorder in the structures caused by photoresist shrinkage, deformation, and roughness of the photoresist surface. The role of disorder has been revealed previously in analysis of angularly-invariant color of Morpho butterfly wing, and its replication in artificial structures[1,2]. On the other hand, for observation in a wider angular range exceeding numerical aperture $\mathrm{NA} \approx 0.5$ colors gradually disappear and the samples appear bright white.

Next, we examine physical origin of the observed colors in the fabricated structures. Detailed theoretical analysis and numerical simulation of their optical properties indicate that a $3 \mathrm{D}$ woodpile architecture photonic crystal with fcc unit cell, refractive index of the rods $n \approx 1.5$, and in plane rod-to-rod spacing $a_{\mathrm{xy}} \approx 800-1000 \mathrm{~nm}$ is not expected to exhibit a fundamental (lowest frequency) PSG at visible wavelengths. In fact, its central wavelength is supposed to be in the NIR spectral range round 1400-1600 nm. These details will be published subsequently. In the meantime, it is helpful to emphasize that $3 \mathrm{D}$ fcc woodpile structures possessing a fundamental PSG at visible wavelengths must have lattice parameter $\mathrm{a}_{\mathrm{xy}} \approx 400 \mathrm{~nm}$ [12], i.e., about twice smaller than in our study. 

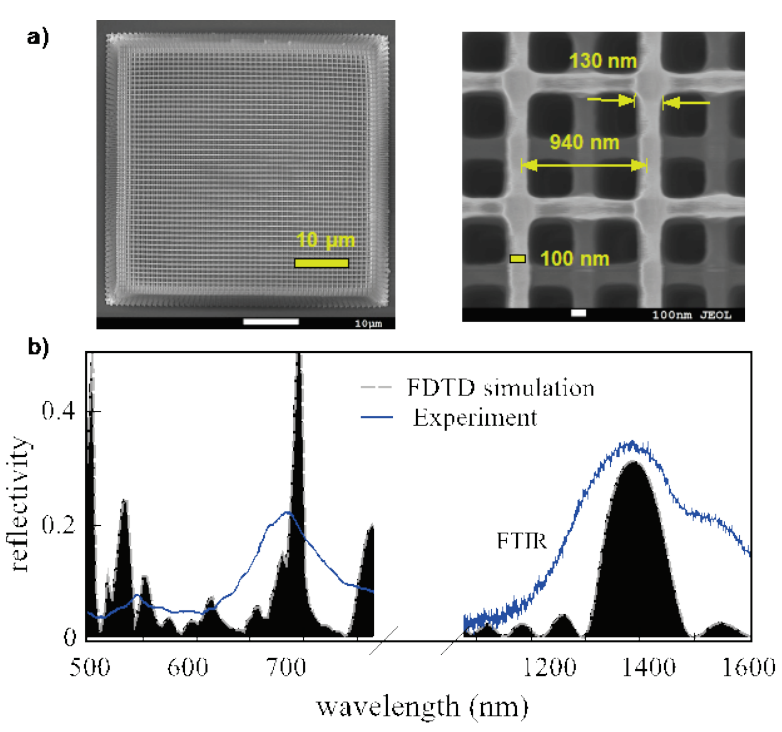

Fig. 3 (a) Top-view SEM images of a woodpile sample with lattice parameters $\mathrm{a}_{\mathrm{xy}}=940 \mathrm{~nm}$ and $\mathrm{d}_{\mathrm{xy}}=130 \mathrm{~nm}$ determined from the image, (b) comparison between measured and numerically simulated visible and NIR reflectivities of the sample shown in (a).

These circumstances suggest that instead of the fundamental PSG, higher-order PSGs located at high normalized frequencies in the photonic band diagram are likely responsible for the observed structural color. Fig. 3 (a) shows SEM images and determination of the actual in-plane lattice parameters, which includes the effects of photoresist shrinkage, to be used in numerical modeling. Using these parameters, and by inferring the corresponding vertical (axial) parameters from the fixed relations outlined earlier, we have performed Finite-Difference Time-Domain (FDTD) simulations of optical reflectance spectra in the spectral range spanning visible and NIR spectral ranges. The obtained simulated spectrum is shown in Fig. 3(b) along with the experimental data. In the latter, NIR reflectance was measured using FTIR. Since experimentally available measurement ranges of visible and FTIR measurements do not overlap, there is a corresponding break in the wavelength axis. As can be seen, simulations and measurements reveal a pair of spectrally matched reflectance peaks. The short-wavelength peak near $\lambda \approx 700 \mathrm{~nm}$ responsible for the visible color is accompanied by the long-wavelength peak centered at approximately twice longer wavelength $2 \lambda \approx 1400 \mathrm{~nm}$. The wavelength doubling suggests strongly that the former reflectance peak corresponds to the second-order PSG, while the latter peak corresponds to the fundamental PSG.

Woodpile architecture photonic crystals have been known to possess second-order and even higher-order PSGs, and to exhibit the corresponding spectral reflectivity peaks. However, the previous demonstration of multiple higher-order PBGs in woodpile photonic structure[6] could not lead to structural color because of large lattice period and non-fcc unit cell strongly deformed (stretched) along the woodpile layer stacking (vertical) direction, such that ratio between the in-plane and vertical lattice parameters in these structures was $a_{x y} / a_{z}>1$. For an fcc unit cell this ratio is $\sqrt{2 / 4} \approx 0.35$. These circumstances led to the condi- tions which allowed observation of higher-order PSGs, but their central wavelength was at NIR and IR wavelengths[6]. In principle, this wavelength can be tuned to visible spectral region by proportional scaling down size of the deformed unit cell. However, this approach can hardly be used for practical realization of structural color, because downscaling will lead to severe decrease of the lateral lattice parameter $\mathrm{a}_{\mathrm{xy}}$ well below $1 \mu \mathrm{m}$, and very high resolution of the DLW will be required to fabricate such a structure lattice. In this study, second order PSG was spectrally tuned into the visible spectral range by proportional downscaling of the fcc lattice period. Compared to the deformed non-fcc unit cell, fcc symmetry provides a realistic balance between the required values of lateral and longitudinal resolution during the fabrication.

\section{Conclusions}

Realization of structural color via second-order photonic stop gap reflectance in 3D woodpile photonic crystal structures fabricated by femtosecond DLW lithography in photoresist may be important for optical environmental sensing applications. Direct 3D woodpile architecture photonic crystals fabricated in this study are highly porous and can be easily infiltrated by liquids and gases capable of modifying optical response of photonic crystal, including that in vicinity of the visible reflectivity bands. This circumstance is helpful for optical sensing, since it allows visual or spectral monitoring of the sensor's response at visible spectral range using cheap detectors and spectrometers. It is helpful to note that spectral features (e.g., high reflectance/low transmission spectral bands) associated with second or higher-order PSGs are more sensitive to lattice deformations and environmental refractive index modification in comparison to those associated with fundamental PSG. Thus, sensor performance may become enhanced due to these factors. Chemical and physical properties and biocompatibility of organic or hybrid photoresists used for DLW nano-lithography suggest that these photoresists may be suitable candidates for emulation of naturally existing nano-structured protein structures, such as wing scales of blue Morpho butterfly. Recently, remarkable sensitivity of Morpho wing scales to the presence of vapors[13] and strong thermal response[7] were reported using experimental observations of reflectivity spectra and their numerical simulations. In the future it might become possible to replicate these remarkable features in artificially nanofabricated 3D photoresist structures.

\section{Acknowledgments}

This work was partially supported by Amada Fund Grant No. AF-2012215. V.P acknowledges support by project "Promotion of Student Scientific Activities" (VP1-3.1ŠMM-01-V-02-003) from the Research Council of Lithuania funded by the Republic of Lithuania and European Social Fund under the 2007-2013 Human Resources Development Operational Programme's priority

\section{References}

[1] S. Kinoshita, and S. Yoshioka, ChemPhysChem., $6,(2005) 1442$. 
[2] A. Saito, M. Yonezawa, J. Murase, S.Juodkazis, V. Mizeikis, M. Akai-Kasaya, and Y.Kuwahara, J. Nanosci. Nanotechnol., 11, (2011) 2785.

[3] K. M. Ho, C. T. Chan, C. M. Soukoulis, R. Biswas, and M. Sigalas, Solid State Commun., 89, (1994) 413.

[4] D. Joannopoulos, S. G. Johnson, J. N. Winn, and R. D. Meade, "Photonic Crystals: Molding the Flow of Light" (Princeton University Press, Princeton and Oxford, 2008) p. 304.

[5] V. Mizeikis, S. Matsuo, S. Juodkazis, and H. Misawa, "Femtosecond laser microfabrication of photonic crystals," Ch. 10 in "3D Laser Microfabrication", ed. by H. Misawa and S. Juodkazis, Willey-VCH Verlag, 2006) p. 369.

[6] M. Straub, M. Ventura, and M. Gu, Phys. Rev. Lett., 91, (2003) 43901.

[7] A. D. Pris, Y. Utturkar, C. Surman, W. G. Morris, A. Vert, S. Zalyubovskiy, T. Deng, H. T. Ghiradella, and R. A. Potyrailo, Nat. Photon., 6, (2012), 195.
[8] A. Ovsianikov, J. Viertl, B. Chichkov, M. Oubaha, B. MacCraith, I. Sakellari, A. Giakoumaki, D. Gray, M. Vamvakaki, M. Farsari, and C. Fotakis, ACS Nano, 2, (2008) 2257.

[9] D.L. Goldfarb, J.J. de Pablo, P.F. Nealey, J.P. Simons, W.M. Moreau, and M. Angelopoulos., Vac. Sci. Technol. B, 18, (2000) 3313.

[10]H.-B. Sun, T. Tanaka, and S. Kawata, Appl. Phys. Lett., 80, (2002) 3673.

[11]Ovsianikov, A. Gaidukeviciute, M. Oubaha, I. Sakellari, A. Giakoumaki, D. Gray, M. Vamvakaki, M. Farsari, and C. Fotakis, Laser Chem., (2008) 493059

[12]J. Fisher and M. Wegener, Laser Photonics Rev., 7, (2012) 22.

[13]R.A. Potyrailo, H. Ghiradella, A. Vertiatchikh, K. Dovidenko, J. R. Cournoyer, and E. Olson, Nat. Photon., 1, (2007) 123.

(Received: August 2, 2013, Accepted: January 15, 2014) 